\title{
Serrated yielding at cryogenic temperatures in structural components of Wendelstein 7-X
}

\author{
Joris H. H. Fellinger, Victor Bykov and Felix Schauer
}

\begin{abstract}
Structural materials like stainless steels are prone to serrated yielding at cryogenic temperatures. The serration effect is the unstable yielding resulting in a zigzag shape of the stress-strain curve observed in displacement controlled tensile tests. Moreover, a local temperature rise in the plastic zone is observed.

In the current paper, the theories explaining the serration effect are briefly discussed. With a coupled thermal-mechanical FE model of a tensile test set up the influence of the test conditions is demonstrated on the observed shape of the stressstrain curve. The FE model is extended to quantify the temperature rise and significance of the serration effect in critical support structures of the magnet system of W7-X.

For W7-X loading rates the temperature rise due to yielding is shown to be moderate and yielding does not localize. As a simple and safe approach, plastic collapse of critical components to be operated at $4 \mathrm{~K}$ can be judged with a pure mechanical analysis neglecting the thermal-mechanical interaction, using yield curves obtained at $77 \mathrm{~K}$ instead.
\end{abstract}

Index Terms - serrated yielding, intermittent yielding, discontinuous flow, cryogenic material properties, coupled thermal-mechanical FEA, W7-X

\section{INTRODUCTION}

$\mathrm{T}$ HE Wendelstein 7-X (W7-X) modular stellarator is under construction at the Max-Planck-Institut für Plasmaphysik in Greifswald, Germany. The W7-X magnet system comprises 70 superconducting coils supported by a massive stainless steel structure to bear the high electromagnetic loads (EML).

The operational limits of the magnet system are defined among other criteria - by the plastic load bearing capacity of various structural components. The superconducting coils are operated at cryogenic temperatures of about $4 \mathrm{~K}$ at which stainless steel (SS) and other alloys show a strong increase of the yield properties compared to room temperature.

However, most alloys exhibit unstable serrated yielding in tensile tests at cryogenic temperatures. Low temperature serrated yielding, also called intermittent yielding or discontinuous flow, is manifested as repetitive staggered stress drops in tensile tests resulting in a zigzag shape of the stressstrain curve, see for instance Fig. 1. The amplitude of the stress drops shows large scatter. Also a local temperature rise

Manuscript received 12 September 2011. (Write the date on which you submitted your paper for review.)

J.H.H. Fellinger, V. Bykov and Felix Schauer are with the Max-PlanckInstitut für Plasmaphysik, Wendelsteinstrasse 1, 17491, Greifswald, Germany phone: +49 383488 2717; fax: $+493834 \quad 88$ 2439; e-mail: joris.fellinger@ipp.mpg.de. in the plastic zone is observed. The plastic zone locally warms up due to dissipation of plastic work into heat, leading to a reduction of the yield limit. Once the yield limit drops, unstable localization of plastic deformation occurs with elastic unloading of the surrounding material, amplifying the plastic straining.

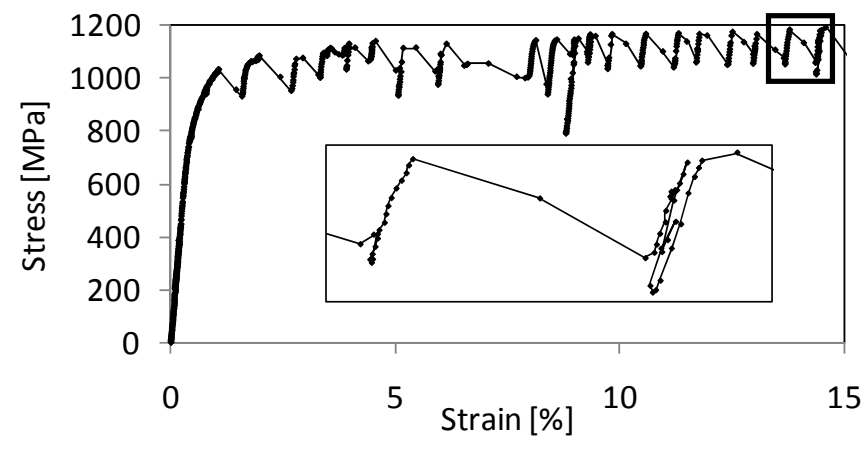

Fig. 1 Example of serrated yielding of stainless steel EN 1.3960

First the serration effect in a tensile test was successfully simulated through coupled thermal mechanical finite element analyses (FEA) and the influence of test conditions was demonstrated. Second, it was shown that the strength drop and local temperature rise for critically loaded components of the magnet structure of W7-X is moderate and can be safely approximated with yield curves derived at $77 \mathrm{~K}$ instead.

\section{THEORETICAL BACKGROUND}

Pustovalov [1] gives an extensive overview of the practical manifestations of low temperature serrated yielding and the theoretical hypotheses. Basinkski [2], [3] postulates that the serration effect results from thermal-mechanical instability, i.e. a competition between thermal softening (TS) and strain hardening (SH) during plastic flow. TS is caused by heating of plastic zone due to conversion of plastic work into heat. Due to the low thermal conductivity and heat capacity of metals at cryogenic temperature, the temperature rise can be calculated from the plastic work for adiabatic boundary conditions as

$$
\rho c_{p} \dot{T}=\eta f_{y} \dot{\varepsilon}_{p l}
$$

with $\eta$ the plastic work conversion factor (between 0-1), $\rho$ the density, $c_{p}$ the specific heat, $f_{y}$ the yield limit, $\dot{T}$ the temperature rise and $\dot{\varepsilon}_{p l}$ the plastic strain rate.

The yield limit is a function of plastic strain and temperature, i.e.

$$
\dot{f}_{y}=\frac{\partial f_{y}}{\partial \varepsilon_{p l}} \dot{\varepsilon}_{p l}+\frac{\partial f_{y}}{\partial T} \dot{T}
$$


Substitution of (1) into (2) leads to

$$
\dot{f}_{y}=Q \dot{\varepsilon}_{p l}
$$

with

$$
Q=\frac{\partial f_{y}}{\partial \varepsilon_{p l}}+\frac{\partial f_{y}}{\partial T} \frac{\eta f_{y}}{\rho c_{p}}
$$

Since the plastic strain increment is always positive, TS dominates $\mathrm{SH}$ if $Q<0$. In Fig. 2, $Q=0$ is plotted for stainless steel EN 1.4429, assuming $\eta=1$, the heat capacity as best fit function for austenitic 18-14 \% nickel-chrome steel from [4], and fitting measured yield curves to a Ramberg-Osgood curve $f_{y}=\left(f_{T 0}+f_{T 1} T+f_{y T 2} T^{2}\right)\left(\varepsilon_{p l} / \varepsilon_{0}\right)^{1 / n}$ with $f_{T 0}=1040 \mathrm{MPa}$, $f_{T 1}=-4 \mathrm{MPa} / \mathrm{K}, f_{T 2}=8 \cdot 10^{-3} \mathrm{MPa} / \mathrm{K}^{2}, \quad n=13.6$ and $\varepsilon_{0}=0.2 \%$. It shows that no serration occurs for low plastic strain (due to the strong $\mathrm{SH}$ ) which is also found in experiments, see for instance Fig. 1.

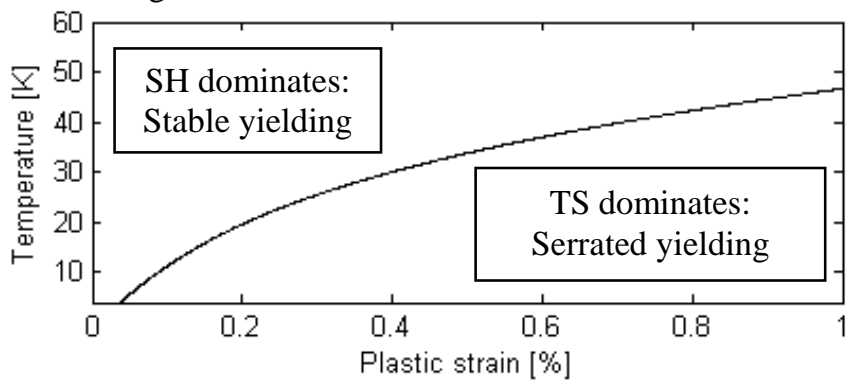

Fig. 2. Onset of serration based on competition between TS and SH

Seeger [5] postulates that serration is due to the breakdown of strong obstacles holding piles-up of dislocations. Obst and Nyilas [6] more specifically claim that serration is dominated by motion of edge rather than screw dislocations and conclude that serration is stopped above the transition temperature where screw dislocations are the main driver of plastic flow. The highest transition temperature for structural materials is around $35 \mathrm{~K}$.

Whichever hypothesis is right, the sensitivity of serrated yielding decreases at higher temperature.

\section{TENSILE TEST SIMULATION}

Whether softening results in serrated deformation depends on the loading conditions: In a load controlled tensile test, no new equilibrium can be found once softening occurs and direct rupture will follow. In a deformation controlled test (the default load condition), softening leads to elastic unloading of the specimen outside the plastic zone and the testing machine, i.e. a localization of deformation occurs. The strain rate (and consequently the temperature rise) in the localized plastic zone is determined by the elastic unloading waves that start to propagate from both ends of the plastic zone into the specimen and the testing machine. The elastic unloading continues until both stress waves encounter each other somewhere in the testing machine. Afterwards, oscillations will develop that depend on the eigenfrequencies of the testing machine without further net elastic unloading. Through a lumped mass-spring analogy, the oscillations can be predicted, see e.g. [7]. As a rough estimate, the longitudinal stress wave speed through a testing machine made of steel is $5 \cdot 10^{3} \mathrm{~m} / \mathrm{s}$ and the length scale of the testing machine is between $0.2-2 \mathrm{~m}$, so dynamic unloading determines the plastic strain rate during the first $4 \cdot 10^{-5}-4 \cdot 10^{-4} \mathrm{~s}$. Afterwards oscillations become relevant and beyond a certain time scale, adiabatic boundary conditions are no longer appropriate as heat flow effects become significant.

To account for the dynamic and transient effects, a coupled thermal-dynamic FE model of a simplified tensile test set up was developed using the explicit solver of Abaqus v6.11, see Fig. 3. The mesh is as coarse as possible to reach practical calculation times for a sensitivity study. The load is applied through the expansion of the elastic actuator. The ambient temperature and top and bottom surface of the specimen are kept at the initial temperature. The remaining outer surface of the specimen is cooled to the ambient by convection.

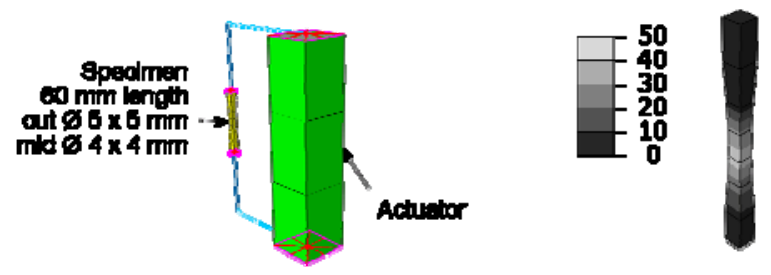

Fig. 3 Mesh of specimen in test frame with highlighted couplings (left) and example temperature distribution of deformed specimen (right)

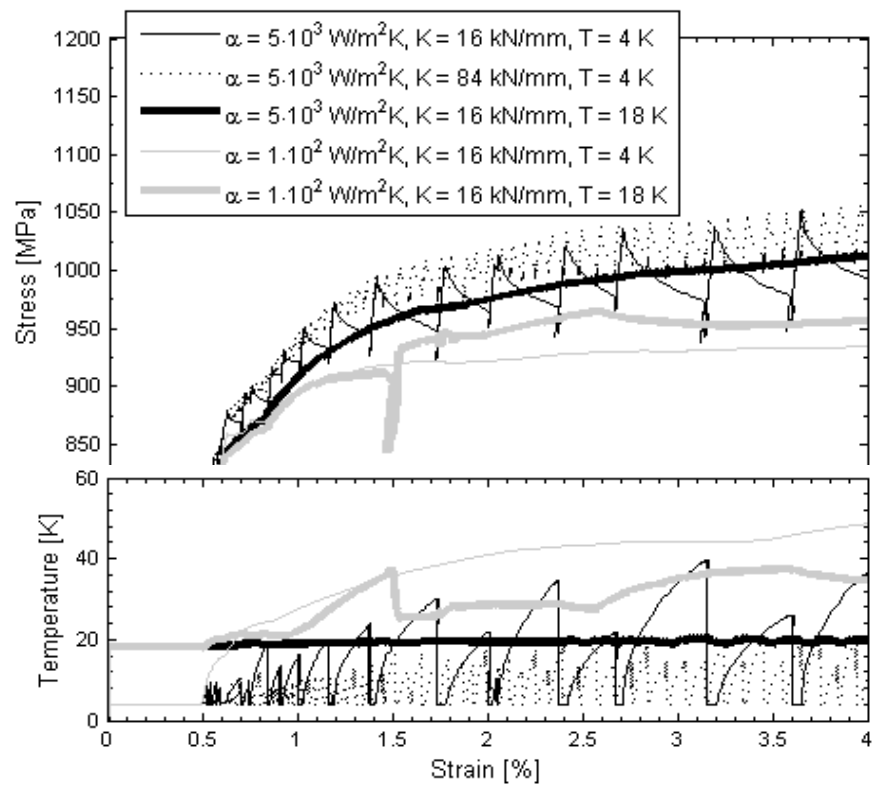

Fig. 4 Simulated stress-strain curve over measurement length of $20 \mathrm{~mm}$ depending on convection, temperature of coolant, and frame stiffness

The model shows that serrated yielding occurs at given loading rate within a certain range of cooling, see Fig. 4. For default liquid cooling with estimated convection of $5000 \mathrm{~W} / \mathrm{m}^{2} \mathrm{~K}$, the simulated curve matches the experimental one Fig. 1 quite well. For slower cooling rates $\left(100 \mathrm{~W} / \mathrm{m}^{2} \mathrm{~K}\right.$ @4K), the temperature in the plastic zone does not return to the initial temperature after the first stress drop and reaches temperatures where no further serration can occur. Consequently, the stress-strain curve becomes smooth. For the faster cooling rates at higher ambient temperature, the temperature spikes and stress drops become ever smaller until also a smooth stress-strain curve is obtained (5000 W/m² K @ $18 \mathrm{~K})$. This tendency was also found experimentally [8]. The 
cooling rate at which serration occurs also depends on the applied strain rate.

Furthermore, the amplitude of the stress drops and temperature spikes depends on the size and stiffness of the testing machine. Notably, the testing machine stiffness $K$ matches that of the test testing machine of Fig. 1. For higher stiffness, the stress drops become smaller in size but occur more frequent.

For slow cooling rate $\left(100 \mathrm{~W} / \mathrm{m}^{2} \mathrm{~K} @ 4 \mathrm{~K}\right)$, the simulation was redone using the standard solver in Abaqus considering coupled thermal mechanical behavior but neglecting dynamic effects. The obtained stress strain curve and temperature development is very similar to the explicit dynamic simulation. So, the standard solver is able to capture the temperature rise for slow cooling rates.

In W7-X, the magnet system is contained in the vacuum of the cryostat, so there is no cooling by convection. As a practical consequence the standard implicit solver of Abaqus can be used instead of the explicit solver, which allows for dense meshes and slow loading rates without dramatic increase of the calculation times.

\section{CRITICAl COMPONENTS OF WENDELSTEIN 7-X}

\section{A. Monolithic steel block loaded by torque}

As a first example, a monolithic stainless steel block bridging two coils of adjacent modules of the magnet system was modeled. With the elastic global model (GM) of the magnet system [9] the load on the block caused by the electromagnetic forces was determined. The main load component, i.e. the torque, was applied on the block in 30 minutes, which is an upper bound for the loading rate in W7-X. Conservatively no cooling was assumed. Simple bilinear elastic-plastic behavior was assumed interpolating $f_{y}=1000,900$ and $800 \mathrm{MPa}$ at 4, 14 or $104 \mathrm{~K}$ respectively, $E=200 \mathrm{GPa}$, Poisson's ratio $v=0.3, \rho=8000 \mathrm{~kg} / \mathrm{m}^{3}, \eta=$ 0.9 (similar to [10]) and thermal properties of TABLE I.

TABLE I: THERMAL PROPERTIES OF STAINLESS STEEL (SS) AND ALUMINIUM-BRONZE (ALUBR)

\begin{tabular}{lcccccccc}
\hline \hline Temperature [K] & material & 4 & 10 & 20 & 40 & 50 & 77 & 100 \\
\hline Specific & SS [11] & 2 & 5 & 10 & & & 200 & \\
heat [J/kgK] & AluBr [12] & 0.4 & 1 & 8 & 60 & & 200 & \\
\hline Thermal & SS [11] & 0.5 & 0.8 & 2 & & 7 & & 10 \\
conductivity [W/mK] & AluBr [12] & 4 & 10 & 20 & & & 60 & 100 \\
\hline \hline
\end{tabular}

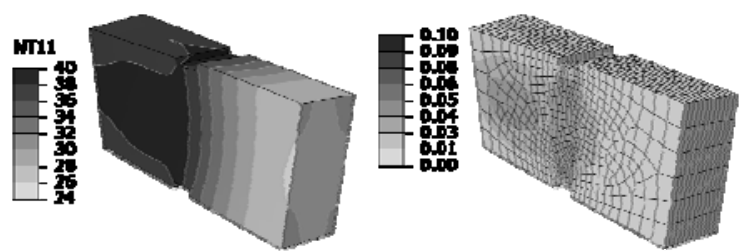

Fig. 5 Temperature [K] (left) and plastic strain [-] (right) after 30 minutes

The temperature field and plastic strain distribution after 30 minutes are shown in Fig. 5. The stress and temperature history is shown in Fig. 6. Despite the symmetry of the model, the plastic strain and temperature fields are asymmetric, attributed to the self enhancing effect of plastic strain and heating. The temperature increase remains limited to $40 \mathrm{~K}$. Due to the temperature increase, the highest loaded area softens but no failure occurs because the stress is redistributed to less loaded zones of the cross section.

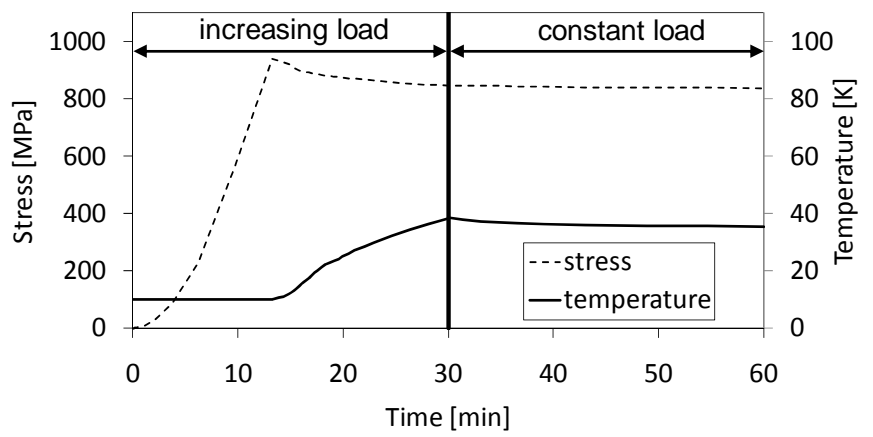

Fig. 6 Temperature and stress development in stainless steel block of the element reaching the highest temperature

\section{B. Narrow support elements}

Hereafter two narrow support elements (NSEs) were modeled, see Fig. 7. NSEs are contact elements with an aluminum-bronze (AluBr) pad with low friction coating in a stainless steel pad frame. The NSEs are mainly loaded under compression allowing for sliding between two coils.

NSE labeled 1E2-1E2 was loaded by a movement normal to the sliding surface (z-direction) until failure. The NSE 1E2$1 \mathrm{E} 2$ was simulated twice using imposed displacement rates of approximately 12 and 1 times the default loading rate of W7-X respectively. Two more reference runs were done without coupled thermal effects assuming constant temperature of 4 or $77 \mathrm{~K}$.

NSE 1E1-1E3 was modeled as a submodel of the GM, in which the cut edges of the coil follow the displacements extracted from the GM. The EML in the GM simulation were scaled up to five times the nominal value in order to be able to load the NSE in the submodel up to collapse.

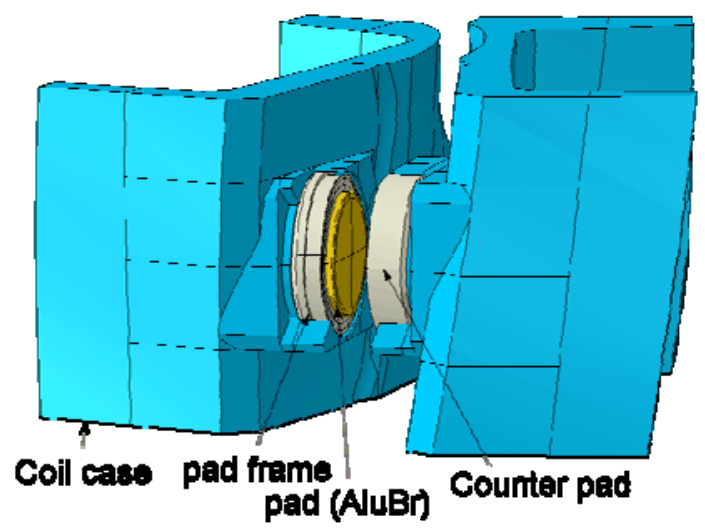

Fig. 7 Model of NSE 1E2-1E2. The separation between the coils is increased and the shoulder of the coil around the pad frame and counter pad is partially suppressed to make the pads and pad frame visible. 


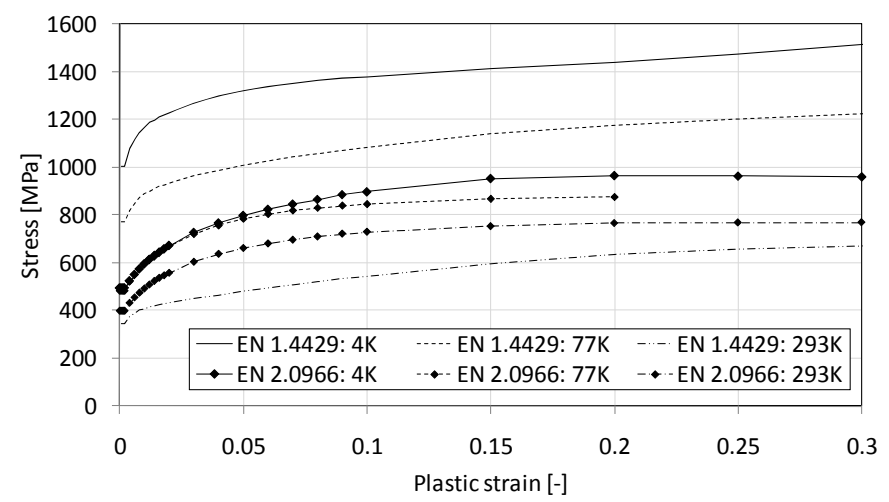

Fig. 8 Experimental stress-strain curves for SS (EN 1.4429) and AluBr (EN 2.0966) in which stress drops have been eliminated

Since the coolant in W7-X is far away from the NSEs, no cooling is considered in the models. In both models, contacts exist between pad and counter surface, pad and pad frame, and pad frame and coil case. In accordance with [13], the thermal contact conductance is assumed to be proportional to the thermal conductivity times the pressure to yield limit ratio.

The yield limit of SS and AluBr are the mean values of stress-strain curves derived in tests in the course of construction of W7-X, with linear interpolation between 4 and 77 and $293 \mathrm{~K}$, see Fig. 8. The thermal properties are given in TABLE I.

The failure load and maximum attained temperature for the NSE 1E2-1E2 are given in Fig. 9. The failure load predicted with $77 \mathrm{~K}$ yield properties is lower than predicted with the coupled approach. The maximum temperature clearly remains below 77K. Also NSE 1E1 1E3 reaches just 27K even under EML scaled by a factor 5. Moreover, no localization of deformation occurs and the plastic strain is moderately distributed, see Fig. 10.

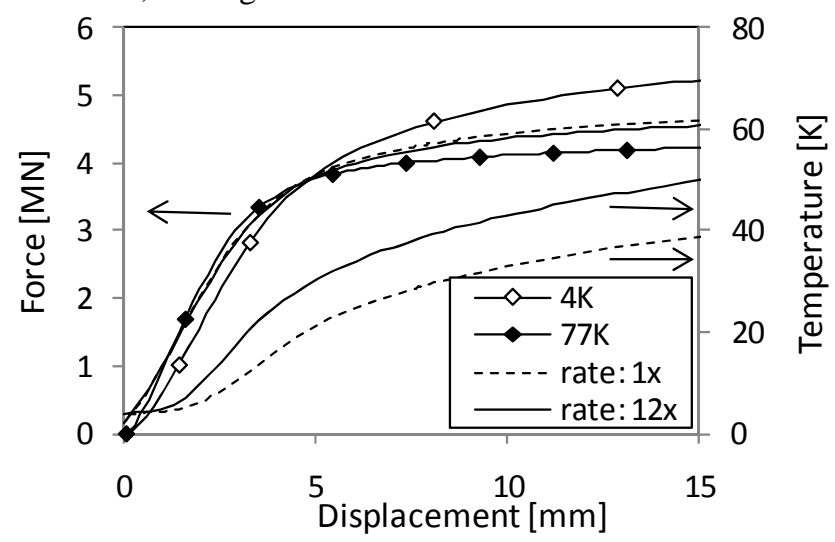

Fig. 9 Contact force development in static and coupled thermal-mechanical analyses of NSE 1E2-1E2and maximum temperature in coupled analyses

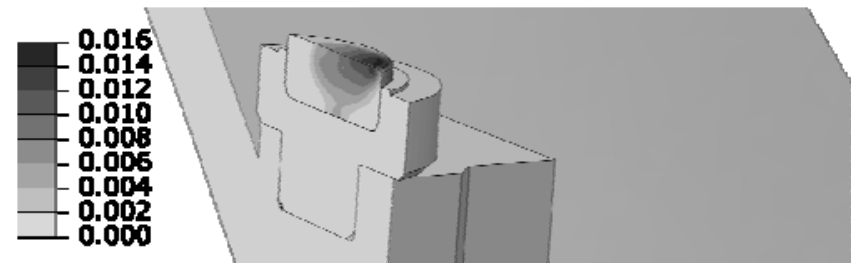

Fig. 10 Plastic strain in section of NSE 1E1-1E3 model at nominal load of 3T

\section{CONCLUSIONS}

The sudden temperature rise and plastic straining as observed in tensile tests during serrated yielding can be predicted by consideration of the equilibrium between the yielding of the plastic zone and the unloading of the elastic part of the test set up.

With a coupled thermal-dynamic model serrated yielding is shown to occur only at moderate cooling conditions. Worse cooling leads to a permanent temperature increase up to a level where no serration takes place. Better cooling leads to such a rapid return to the initial temperature that no stress drops are observed, see Fig. 4. For lower initial temperatures, the specimen is more prone to serration. The size of the stress drops increase for lower testing machine stiffness.

For the magnet system of W7-X, loaded by a coil activation rate up to nominal operation in 30 minutes, a gradual temperature increase is found. Since critical cross sections are not uniformly loaded, stress redistribution within the cross section is possible once yielding occurs. Opposite to a tensile test no localization of deformation in the cross section develops.

According to the FEA, the temperature rise of critical components of W7-X at plastic collapse is limited to some $40 \mathrm{~K}$ even without consideration of cooling. The plastic collapse can thus conservatively be determined assuming yield properties measured at $77 \mathrm{~K}$.

\section{REFERENCES}

[1] V.V. Pustovalov, "Serrated deformation of metals and alloys at low temperature (Review)", in Low temperature physics, Vol. 34, No. 9, 2008, pp. 683-723

[2] Z.S. Basinski, "The instability of plastic flow of metals at very low temperatures, part I”, Proceedings Royal Society A240, 1956, pp.229242

[3] Z.S. Basinski, "The instability of plastic flow of metals at very low temperatures, part II”, Australian Journal of Physics, Vol. 13, 1960, pp. 354-358

[4] J.M. Corsan, N.I. Mitchem, "The specific heat of 15 stainless steels in the temperature range of 4K-30K", in Cryogenics 01-1979, pp. 11-16

[5] A. Seeger, "The mechanisms of glide and work hardening in f.c.c. and h.c.p. metals", in Dislocations and mechanical properties of crystals, Ed. J.C. Fisher et al., NY: Wiley, 1957, pp 243-330

[6] B. Obst, A. Nyilas, "Experimental evidence on the dislocation mechanism of serrated yielding in f.c.c. metals and alloys at low temperatures", in Materials Science and Engineering, A137, 1991, pp. 141-150

[7] G.Y. Chin, W.F. Hosford Jr. and W.A. Backofen, "Influence of the mechanical loading system on low temperature plastic instability", in AIME Trans. Metallurgical Soc., Vol. 230, 08-1964, pp 1043-1048

[8] E.M. Medvedev, "On the characteristic of low temperature discontinuous plastic deformation of metals", in Cryogenics, 09-1976, pp. 533-536

[9] V. Bykov, F. Schauer, K. Egorov, P.van Eeten, J. Fellinger, M. Sochor, N. Jaksic, A. Tereshchenko, A. Dübner, A. Dudek, D. Zacharias, D. Hathiramani, P. Czarkowski, Q. Yang, "Structural analysis of W7-X: From design to assembly and operation (accepted for publication)", in Fus. Eng. Des., to be published

[10] K. Shibata, T. Fujita, "Serration of Fe-Ni stainless steels at very low temperatures and its computer simulation" in Transactions ISIJ, Vol. 26, 1986, pp. 1065-1072

[11] R.P. Reed, A.F. Clark, Materials at low temperature, American Society for Metals, 1983

[12] H.J. Hucek, Wilkes, K.R. Hanby and J.K Thompson, Handbook on materials for superconducting machinery, NTIS, 1977

[13] M.G. Cooper, B.B. Mikic, M.M. Yovanovisch, "Thermal contact conductance”, in Int. Jrnl of heat transfer, Vol. 12, 1969, pp. 279-300 